\title{
Role of Retinal Pigment Epithelial Cell $\beta$-Catenin Signaling in Experimental Proliferative Vitreoretinopathy
}

\author{
Kazuhiko Umazume, ${ }^{*}$ Rintaro Tsukahara, ${ }^{*}$ LanHsin Liu, ${ }^{*}$ Juan P. Fernandez de Castro, ${ }^{*}$ Kevin McDonald, ${ }^{*}$ Henry J. Kaplan, ${ }^{*}$ \\ and Shigeo Tamiya ${ }^{* \dagger}$
}

From the Departments of Ophthalmology and Visual Sciences* and Biochemistry and Molecular Biology, ${ }^{\dagger}$ University of Louisville, Louisville, Kentucky

\author{
Accepted for publication \\ January 15, 2014 \\ Address correspondence to \\ Shigeo Tamiya, Ph.D., 301 \\ E. Muhammad Ali Blvd, \\ Louisville, KY 40202. E-mail: \\ shigeo.tamiya@louisville.edu.
}

\begin{abstract}
Proliferative vitreoretinopathy is caused by the contraction of fibrotic membranes on the epiretinal surface of the neurosensory retina, resulting in a traction retinal detachment and loss of visual acuity. Retinal pigment epithelial (RPE) cells play an important role in formation of such fibrotic, contractile membranes. We investigated the role of Wnt/ $\beta$-catenin signaling, a pathway implicated in several fibrotic diseases, in RPE cells in proliferative vitreoretinopathy. In vitro culture of swine RPE sheets resulted in nuclear translocation of $\beta$-catenin in dedifferentiated RPE cells. FH535, a specific inhibitor of $\beta$-catenin signaling, reduced the outgrowth of cultured RPE sheets and prevented dedifferentiated RPE cell proliferation and migration. It also inhibited formation of contractile membranes by dedifferentiated RPE cells on collagen I matrices. Expression and function of the $\beta$-catenin signaling target connexin-43 were down-regulated by FH535, and functional blockade of connexins with carbenoxolone also prevented the in vitro formation of fibrotic, contractile membranes. Intravitreal injection of FH535 in swine also inhibited formation of dense, contractile membranes on the epiretinal surface and prevented development of traction retinal detachment. These findings demonstrate that $\beta$-catenin signaling is involved in formation of contractile membranes by dedifferentiated RPE cells and suggest that adjunctive treatment targeting this pathway could be useful in preventing proliferative vitreoretinopathy. (Am J Pathol 2014, 184: 1419-1428; http://dx.doi.org/10.1016/j.ajpath.2014.01.022)
\end{abstract}

Organs respond to insults or damage by activating wound healing mechanisms. However, a persistent and/or deregulated wound response leads to fibrosis, which in turn often leads to organ dysfunction, with detrimental consequences. ${ }^{1}$ Proliferative vitreoretinopathy (PVR) is a fibrotic complication caused by aberrant wound healing after rhegmatogenous retinal detachment surgery or posterior segment ocular trauma. ${ }^{2-4}$ The hallmark of PVR is the development of a dense fibrotic contractile epiretinal membrane composed of extracellular matrix and a variety of cell types. Analyses of epiretinal membranes from patients, as well as animal models of experimental PVR, have identified retinal pigment epithelial (RPE) cells, glial cells, immune cells, fibroblasts, and myofibroblasts. ${ }^{4-7}$ Experimental evidence shows that RPE and glial cells, both of which contribute to the final outcome of PVR, ${ }^{4-10}$ can dedifferentiate and transform into myofibroblasts, the major contractile-cell phenotype found in epiretinal membranes. $4,11,12$

In the present study, we focused on RPE cells because they play an important role during the early stages of PVR development. ${ }^{4,13-15}$ On release from their monolayer, RPE cells undergo epithelial-mesenchymal transition (EMT) and dedifferentiate to assume a mesenchymal morphology. ${ }^{6,12,16-19}$ Dedifferentiated RPE cells can migrate, proliferate, and transform into myofibroblasts. Our research group has previously reported that expression of cyclin D1, a major $\beta$-catenin/Wnt signaling target, ${ }^{20}$ is significantly increased in dedifferentiated RPE cells. ${ }^{16}$ Given that aberrant activation of canonical Wnt signaling has been reported in other fibrotic diseases, ${ }^{1,21}$ in the present study we investigated the role of this pathway in development of PVR. We tested effects of the Wnt/ $\beta$ -

Supported by grant DM090475 from the Department of Defense, a University of Louisville Clinical \& Translational Science pilot grant, and Kentucky Science and Engineering Foundation R\&D Excellence grant KSEF-148-502-10-271 (S.T.); and by Research to Prevent Blindness the Kentucky Lions Eye Foundation, and the Kentucky Research Challenge Trust Fund (H.J.K.).

Disclosures: None declared. 
catenin signaling inhibitor FH535 $5^{22,23}$ in both in vitro and in vivo PVR models, after confirmation of active $\mathrm{Wnt} / \beta$ catenin signaling in dedifferentiated RPE cells.

\section{Materials and Methods}

\section{Isolation of RPE Sheets for Primary Culture in Vitro}

Cell culture supplies were from Life Technologies (Carlsbad, CA), unless stated otherwise. Porcine RPE sheets were isolated from adult eyes, using dispase as described previously. ${ }^{16}$ Isolated RPE sheets were cultured in Dulbecco's modified Eagle's medium (DMEM) supplemented with $10 \%$ fetal bovine serum (FBS) either on porcine posterior lens capsules or in a culture flask. RPE sheets on lens capsules were cultured in the presence or absence of 10 or $20 \mu \mathrm{mol} / \mathrm{L}$ FH535 (EMD Millipore, Billerica, MA), ${ }^{22,23}$ a $\beta$-catenin/TCF signaling inhibitor. Dimethyl sulfoxide (DMSO), used as solvent for FH535, served as a control. The concentration of DMSO did not exceed $0.1 \%$ in any sample. RPE cells in flask were passaged with trypsin and were used at passage 3 for migration and contraction assays and for Western blot analysis.

\section{Analysis of RPE Sheet Enlargement and Cell Proliferation}

Growth (enlargement) of RPE sheets on the lens capsule was assessed as described previously. ${ }^{24}$ Proliferation was assessed by incorporation of bromodeoxyuridine (BrdU) as described previously. ${ }^{16}$ In brief, $\mathrm{BrdU}^{+}$cells were detected using an anti-BrdU monoclonal antibody (clone G3G4; Developmental Studies Hybridoma Bank, Iowa City, IA) and Alexa Fluor 488-conjugated goat anti-mouse secondary antibody (Life Technologies). The ratio of $\mathrm{BrdU}^{+}$cells to total numbers of nuclei was determined using ImageJ software version 1.44p (NIH, Bethesda, MD).

In addition to BrdU incorporation, increase in the number of cultured swine RPE cells at passage 3 was used to assess the effect of FH535 independent of dedifferentiation. For this purpose, passage 3 RPE cells were seeded at $2.0 \times 10^{5}$ per $35-\mathrm{mm}$ dish and were cultured in $10 \%$ FBS-DMEM for 24 hours. The medium was then replaced with 10\% FBS-DMEM containing FH535 or DMSO (control). RPE cells were further cultured for 48 hours. Finally, each dish was trypsinized, and cells were counted using a hemocytometer.

\section{Cell Apoptosis Assay}

Passage 3 RPE cells were cultured as described above to determine the increase in number of cells in the presence or absence of FH535. After 48 hours, cells were fixed with $4 \%$ paraformaldehyde for 10 minutes, and TUNEL staining was performed using a commercially available kit (DeadEnd fluorometric TUNEL system; Promega, Madison, WI). Three separate areas were photographed for each dish, and the ratio of $\mathrm{TUNEL}^{+}$cells to total number of nuclei was determined using ImageJ software version $1.45 \mathrm{~m}$.

\section{Immunocytochemical Staining}

Immunostaining of fixed samples was performed as described previously. ${ }^{16}$ Primary antibodies for anti-active $\beta$-catenin (05-665; Millipore, Billerica, MA), which detects signaling-capable $\beta$-catenin not phosphorylated on S37 and $\mathrm{T} 41,{ }^{25}$ and Alexa Fluor 488-conjugated donkey anti-mouse secondary antibodies (Life Technologies) were used for detection. Nuclei were counterstained with Hoechst 33342 dye. In the absence of primary antibodies (negative control), only faint, negligible staining was observed. Stained and mounted samples were observed using an Olympus (Tokyo, Japan) FV300 confocal microscope.

\section{Migration Assay}

To assess the effect of FH535 on cell migration, the scratch wound assay was performed as described previously. ${ }^{24,26}$ In brief, RPE cells at passage 3 were preincubated for 1 hour with FH535 and then scratched. After scratching, the medium was immediately replaced with $10 \%$ FBS-DMEM containing $5 \mu \mathrm{mol} / \mathrm{L}$ aphidicolin (Sigma-Aldrich, St. Louis, $\mathrm{MO}$ ), used to inhibit cell proliferation ${ }^{27}$ in the presence of FH535 or DMSO (control). Migration of cells into the scratched area was assessed at 24 hours.

\section{In Vitro Contractile Membrane Formation}

Dedifferentiated RPE cells were used in vitro to mimic epiretinal membrane formation and contraction by modifying type I collagen matrix contraction assays. ${ }^{28-30}$ Neutralized type I collagen (BD Biosciences, San Diego, CA) at $2.5 \mathrm{mg}$ / $\mathrm{mL}$ was gelled in 24-well plates. RPE cells at passage 3 in $25 \%$ vitreous fluid-DMEM (without serum) were added to each well at $2.0 \times 10^{5}$ cells per well. Swine vitreous fluid was obtained in a similar manner as that described by Meitinger et al. ${ }^{31}$ After 4 hours, FH535, GW9662 (1508; R\&D Systems, Minneapolis, MN), an analog of FH535 that inhibits PPAR but not $\beta$-catenin signaling, ${ }^{22}$ or carbenoxolone disodium (3096; R\&D Systems) (CBX), a gap junction inhibitor, was added to the medium. DMSO was used for controls. After 3 days of culture, the matrices were gently released from the well and photographed after 4 hours. In some cases, 24 hours of treatment with FH535 or CBX was performed after cells were cultured on the collagen matrix for 3 days. In such cases, the matrices were released on day 4 and were photographed after 4 hours. The area of the matrix was quantified using ImageJ software version $1.45 \mathrm{~m}$.

\section{RNA Interference}

Passage 2 RPE cells at $80 \%$ to $90 \%$ confluence in $25-\mathrm{cm}^{2}$ flasks were transfected with 100 pmol $\beta$-catenin siRNA (6238; 
Cell Signaling Technology, Danvers, MA) and $28 \mu \mathrm{L}$ RNAiMAX reagent in $1 \mathrm{~mL}$ Opti-MEM medium (both from Life Technologies). Control siRNA (6568S; Cell Signaling Technology) at the same concentration served as a control. At 20 hours after transfection, cells were trypsinized and used in the in vitro contractile membrane formation assay.

\section{Scrape Loading and Dye Transfer Assay}

The scrape loading and dye transfer assay was used to assess gap junctional intercellular communication, as described previously. ${ }^{32}$ In brief, $5 \times 10^{4}$ passage $3 \mathrm{RPE}$ cells in $150 \mu \mathrm{L}$ of $10 \%$ FBS-DMEM were seeded onto the middle of 35-mm dishes. After attachment (3 to 4 hours), $1.5 \mathrm{~mL}$ of $10 \%$ FBS-DMEM was added. After 24 hours, the medium was replaced with $25 \%$ vitreous-DMEM in the presence of FH535 or DMSO (control), and the cells were cultured for 3 days. On day 3, cells were rinsed once with Dulbecco's PBS and a confluent patch was scraped with a surgical blade. Cells were immediately exposed to Dulbecco's PBS containing $1 \mathrm{mg} / \mathrm{mL}$ Lucifer Yellow (Life Technologies) for 10 minutes. After removal of Lucifer Yellow-containing buffer and a quick rinse, cells were fixed and photographed using an inverted fluorescence microscope. The distance from the scraped edge to the most distal Lucifer Yellow-positive cell was measured at three different points within each image using Photoshop software version 12.1 (Adobe Systems, San Jose, CA) and was averaged for each dish.

\section{Western Blot Analysis}

Western blot analysis was performed as described previously. ${ }^{16}$ For RPE sheets cultured on lens capsules, the heavily pigmented differentiated RPE cells were microdissected from the less pigmented dedifferentiated RPE cells and were lysed separately in radioimmunoprecipitation assay buffer. Primary cultured RPE cells at passage 3 were seeded on collagen I-coated wells and were cultured for 3 days in $25 \%$ vitreous fluid-DMEM in the presence of FH535 or DMSO before cell lysis. Cells treated with control or $\beta$-catenin siRNA were lysed at the end of the in vitro contractile membrane formation assay. Proteins in lysed samples were resolved by SDS-PAGE, transferred to nitrocellulose membranes, and blotted with primary and infrared dye-conjugated secondary antibodies. Band detection was performed using an Odyssey infrared scanner (LI-COR Biosciences, Lincoln, NE).

\section{$\mathrm{GFP}^{+}$RPE Isolation and Culture}

Fetal $\mathrm{GFP}^{+}$RPE cells from embryonic $\mathrm{GFP}^{+}$swine eyes were isolated and cultured as described previously. ${ }^{33,34}$ In brief, RPE cells and partial sheets isolated using dispase were cultured in tissue culture flasks for 2 to 6 weeks until confluent. The cells were then trypsinized, and cultured on
Transwell filters (Corning Life Sciences, Tewksbury, MA) for a further 3 to 6 weeks to induce RPE cell redifferentiation. Cells isolated and cultured by this method were $>95 \%$ cytokeratin positive. Transwell inserts with cultured RPE cells were treated with dispase to obtain RPE cell clusters, which were used for induction of PVR in vivo.

\section{PVR Induction Surgery}

Swine were used for induction of PVR because their eye size, retinal vasculature, and cone-enriched area centralis are similar to those of the human eye. ${ }^{35}$ PVR was induced in female domestic swine (Oak Hill Genetics, Ewing, IL) as described previously, with slight modification. ${ }^{34}$ In brief, PVR was induced using a three-step procedure involving 25 -gauge pars plana vitrectomy, total retinal detachment by subretinal injection of balanced salt solution (Alcon Laboratories, Fort Worth, TX) in each of the four quadrants using a 41-gauge needle (to mimic a rhegmatogenous retinal detachment), and injection of $8 \times 10^{4} \mathrm{GFP}^{+} \mathrm{RPE}$ cells into the vitreous cavity at the conclusion of the surgical procedure.

\section{Administration of FH535 and Grading of PVR in Vivo}

FH535 was administered at a final estimated concentration of $20 \mu \mathrm{mol} / \mathrm{L}$ by injecting $0.8 \mathrm{mmol} / \mathrm{L}$ FH535 in $0.1 \mathrm{~mL}$ of $2 \%$ DMSO-balanced salt solution into the vitreous cavity through a 30 -gauge needle attached to a $1-\mathrm{mL}$ syringe. For controls, only the $0.1 \mathrm{~mL}$ of $2 \%$ DMSO-balanced salt solution was injected. The concentration of FH535 injected was based on the assumption that the volume of the pig vitreous cavity is approximately $4 \mathrm{~mL},{ }^{35}$ and thus the final concentration of FH535 and of DMSO was estimated to be $20 \mu \mathrm{mol} / \mathrm{L}$ and $0.05 \%$, respectively. FH535 was administered immediately after the induction surgery and on postoperative days 3,7 , and 10 .

Clinical examinations using indirect ophthalmoscopy were performed on postoperative days 3, 7, 10, and 14 by a single ophthalmologist (K.U.) in a masked manner. PVR grading was based on the Silicone Study Classification System for PVR. ${ }^{36}$ A single investigator (K.U.) performed the clinical examinations and evaluated the retinal status in a masked manner.

\section{Histological and Immunohistochemical Procedure}

Animals were euthanized, and eye cups were prepared from enucleated globes and fixed as described previously. ${ }^{34}$ $\mathrm{GFP}^{+}$cells on the surface of the retina were photographed using a dissecting microscope equipped with a fluorescein isothiocyanate excitation filter set (Zeiss MicroImaging, Thornwood, NY; Carl Zeiss Microscopy, Jena, Germany). Paraffin sections were prepared from regions of retina with attached $\mathrm{GFP}^{+}$cells, and immunostaining for GFP was performed as described previously. ${ }^{34}$ 
A

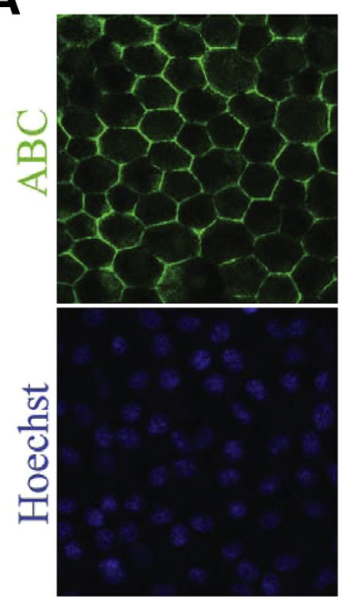

Diff.

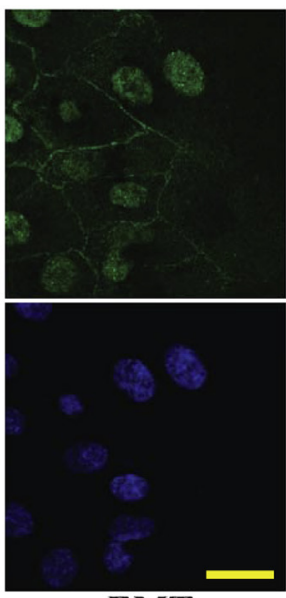

EMT
B
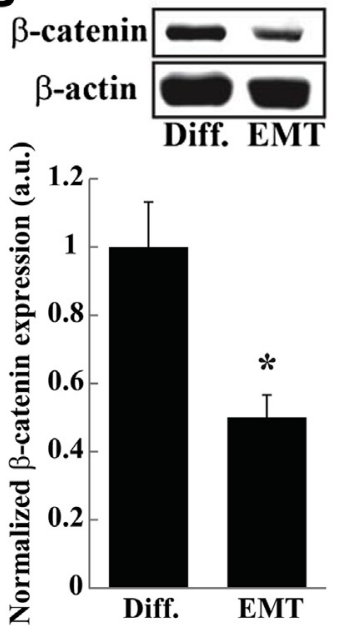

C

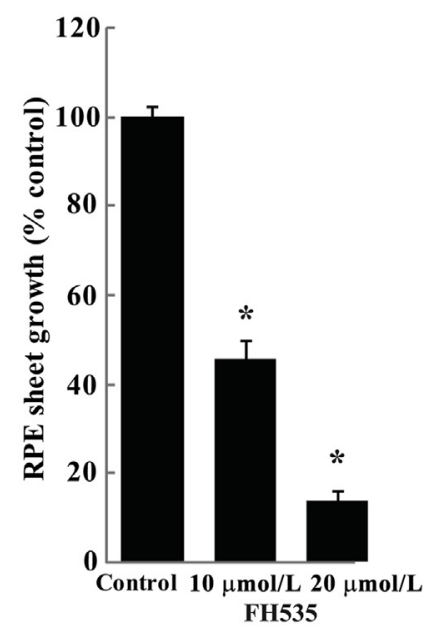

D
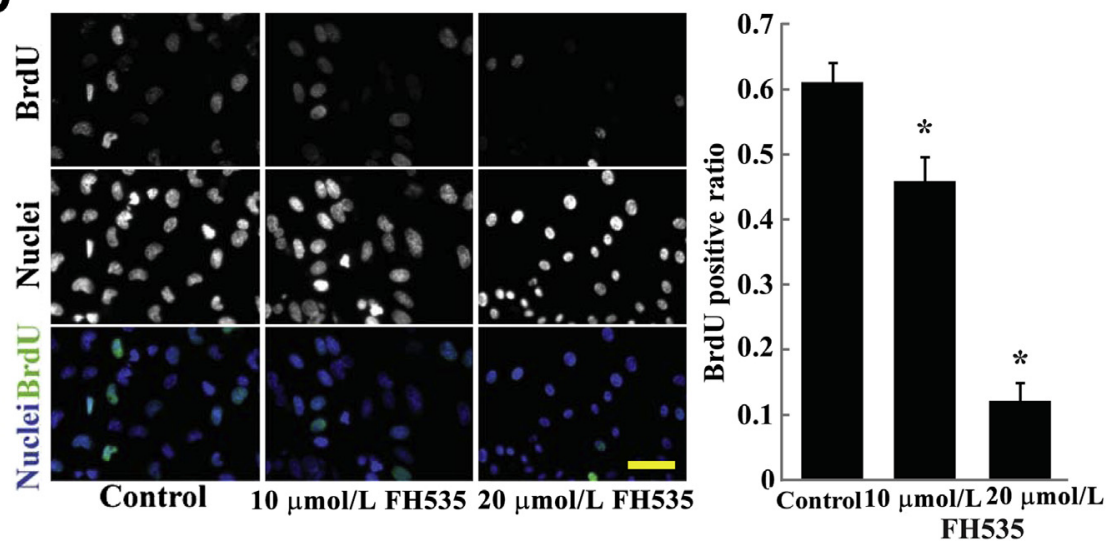

Figure 1 Expression of $\beta$-catenin in RPE cells and the effect of FH535 on cultured RPE sheets. A: Immunocytochemical staining of active $\beta$-catenin. RPE cells cultured for 6 days were fixed and stained using an active $\beta$-catenin antibody (green). Nuclei were counterstained with DAPI (blue). Staining for active $\beta$-catenin was detected in the nucleus of dedifferentiated RPE cells, but not in the nucleus of differentiated cells. B: Western blot analysis of total $\beta$-catenin expression in cultured RPE cells. $\beta$-Catenin expression was significantly reduced in dedifferentiated RPE cells. C: Effect of FH535 on RPE sheet enlargement stimulated by $10 \%$ FBS-supplemented medium. FH535 inhibited RPE sheet enlargement in a concentration-dependent manner. D: Assessment of RPE cell proliferation by BrdU uptake shows concentration-dependent inhibition by FH535. Data are expressed as means \pm SEM of 4 different samples (B) or $\geq 12$ different samples from three different batches (C and D). ${ }^{*} P<0.05$ versus control. Scale bars: $20 \mu \mathrm{m}(\mathbf{A}) ; 100 \mu \mathrm{m}(\mathbf{D})$. ABC, active $\beta$-catenin; Diff, differentiated; EMT, dedifferentiated.

\section{Ethics}

The animal protocol was approved by the University of Louisville Institutional Animal Care and Use Committee and adhered to the Association for Research in Vision and Ophthalmology Statement for the Use of Animals in Ophthalmic and Visual Research.

\section{Statistical Analysis}

The difference in PVR grading was analyzed with $U$-test, and Student's $t$-test was used for analysis of in vitro assays (sheet growth, migration, proliferation, and collagen matrix contraction). MedCalc statistical software version 12.1.1 (MedCalc Software, Ostend, Belgium) was used for statistical analysis. A $P$ value of $<0.05$ was considered statistically significant.

\section{Results}

\section{$\beta$-Catenin Signaling Is Activated in RPE Cells in EMT}

Our research group has previously shown that cells at the edge of cultured RPE sheets undergo dedifferentiation, but that cells within the center of the sheets retain a differentiated phenotype. ${ }^{16}$ Furthermore, dedifferentiated cells have significantly increased expression of cyclin D1, a wellestablished target of $\beta$-catenin signaling. In the present study, we first investigated whether $\beta$-catenin signaling is activated in RPE cells on EMT. Immunohistochemical staining of active $\beta$-catenin revealed translocation to the nucleus in dedifferentiated cells, whereas differentiated cells retained expression at the cell-cell border (Figure 1A). Moreover, examination of total $\beta$-catenin by Western blot revealed significantly lower $\beta$-catenin expression in the dedifferentiated cells (Figure 1B). These results strongly 
suggested that $\beta$-catenin signaling is activated in RPE cells during or after EMT.

\section{Effect of $\beta$-Catenin Signaling Inhibition on RPE Cells in Vitro}

Effect on RPE Sheet Enlargement, Proliferation, and Migration

FH535, an inhibitor of $\beta$-catenin signaling, was used to investigate the role of this signaling pathway in RPE cell changes related to PVR. We investigated the effect of FH535 on RPE sheet enlargement, which involves EMT, migration, and proliferation. FH535 inhibited RPE sheet enlargement in a concentration-dependent manner; $20 \mu \mathrm{mol} / \mathrm{L}$ FH535 prevented sheet enlargement by $>80 \%$ (Figure 1C). Furthermore, BrdU incorporation analysis demonstrated that proliferation also was inhibited by FH535 in a concentrationdependent manner (Figure 1D).

Next, we investigated the effect of FH535 on proliferation and migration independent of EMT by using passage $3 \mathrm{RPE}$ cells, which have already undergone dedifferentiation. FH535 inhibited the increase in cell number over a 3-day culture period in a concentration-dependent manner (Figure 2A) with little effect on cell apoptosis (Figure 2B), demonstrating that the effect on proliferation is independent of its effect on EMT. Furthermore, Western blot analysis revealed that FH535 treatment significantly reduced expression of cyclin D1 in dedifferentiated RPE cells (Figure 2C). Separate from proliferation, migration of RPE cells was also significantly inhibited in the presence of FH535 compared with controls (Figure 2D).

Effect on Contractile Membrane Formation

The formation and contraction of the epiretinal membrane is the hallmark of PVR. Contraction of this multicellular membrane plays an important role in traction retinal detachment (TRD) during PVR. We have developed an assay to examine formation of a contractile membrane in vitro using dedifferentiated RPE cells. Under control conditions, vitreous-supplemented medium induced formation of a dense membrane on the surface of a collagen matrix (Figure 3A). Matrices contracted swiftly upon release from the plate (shrinking by $49 \%$ on average) (Figure 3B). In the presence of FH535, however, formation of the dense membrane was inhibited. Collagen matrix contraction was reduced significantly in a concentration-dependent manner by FH535, with matrix contraction $<10 \%$ of that of the control in cells treated with $20 \mathrm{mmol} / \mathrm{L}$ FH535 (Figure 3B).

Because FH535 has been reported to inhibit PPAR signaling as well as $\beta$-catenin signaling, ${ }^{22}$ we also investigated the effect of GW9662 (an analog of FH535 that inhibits PPAR signaling but not $\beta$-catenin signaling) using the contractile membrane formation assay. In contrast to FH535, GW9662 had no significant effect on matrix contraction (Figure 3C). The importance of $\beta$-catenin signaling in contraction was further confirmed by knocking down $\beta$-catenin expression by siRNA. Reduction in $\beta$-catenin protein expression by siRNA treatment was accompanied by significant reduction in matrix contraction (Figure 3, $\mathrm{D}$ and $\mathrm{E})$.

Next, we investigated molecules downstream of $\beta$-catenin signaling that are involved in formation of the dense contractile membrane. Expression of connexin-43, a wellestablished target of $\beta$-catenin signaling, ${ }^{37}$ was significantly reduced when dedifferentiated RPE cells were cultured in the presence of FH535 (Figure 4A). The scrape loading and dye transfer assay also revealed inhibition of gap junction function; the distance of Lucifer Yellow diffusion in FH535-treated cells was significantly shorter than in controls (Figure 4B). The involvement of connexin function in
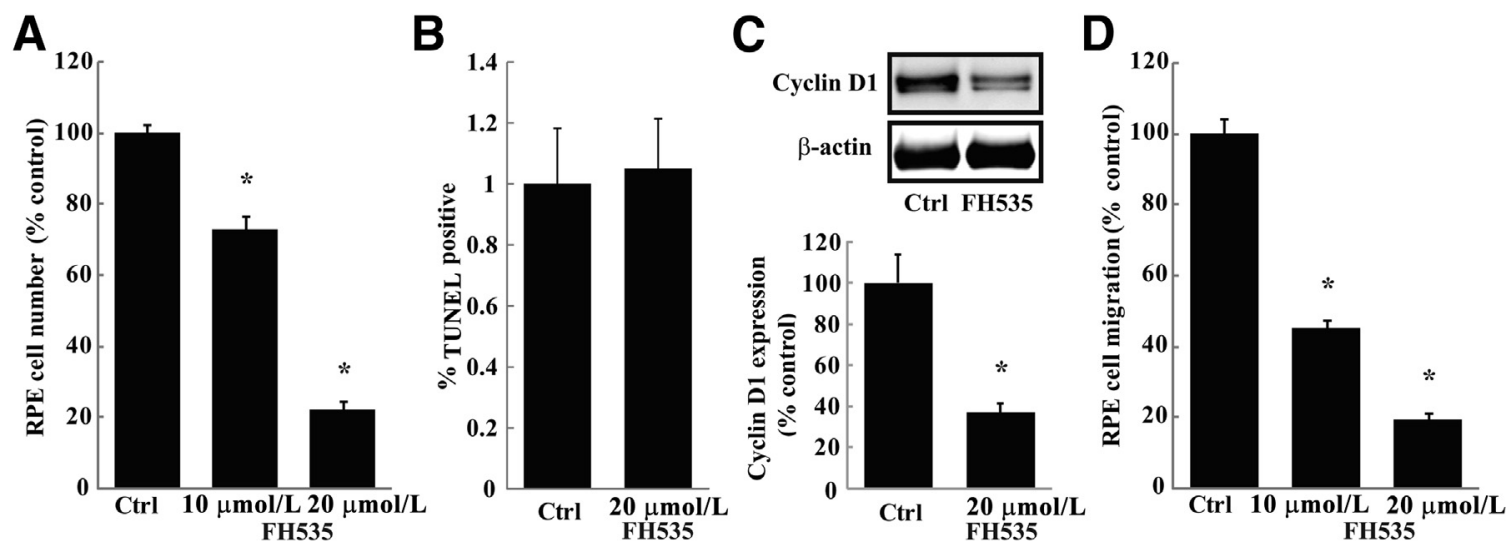

Figure 2 Effect of FH535 on dedifferentiated passage 3 RPE cells. A: Dedifferentiated RPE cells were cultured for 48 hours in the presence or absence of FH535 in 10\% FBS-supplemented medium. FH535 prevented the increase in cell number in a concentration-dependent manner. B: TUNEL assay analysis of apoptosis of dedifferentiated RPE cells cultured for 48 hours in the presence or absence of FH535 in $10 \%$ FBS-supplemented medium. There was no difference between $20 \mu \mathrm{mol} / \mathrm{L}$ FH535 and control. C: Western blot analysis of cyclin D1 expression. Treatment with $20 \mu \mathrm{mol} / \mathrm{L}$ FH535 significantly reduced cyclin D1 expression. D: Migration was assessed by scratch wound assay in the presence or absence of FH535. FH535 inhibited migration in a concentration-dependent manner. Data are expressed as means \pm SEM of three different experiments with triplicates for each experiment (A and $\mathbf{D})$ or of 4 (C) or 8 (B) different samples. ${ }^{*} P<0.05$ versus control (Ctrl). 
A

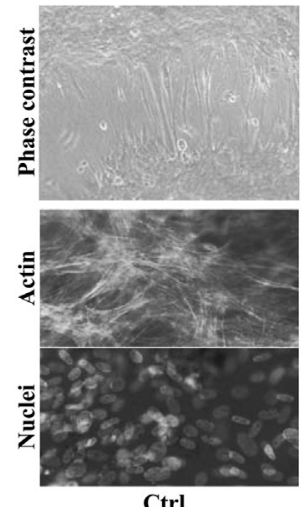

Ctrl

C

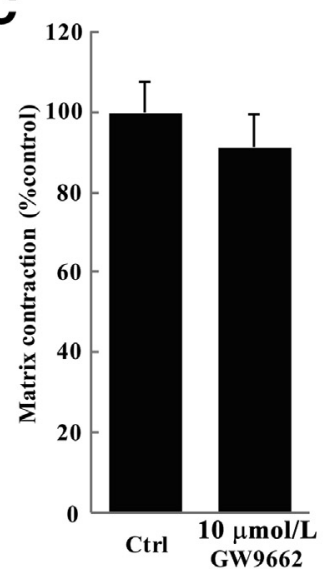

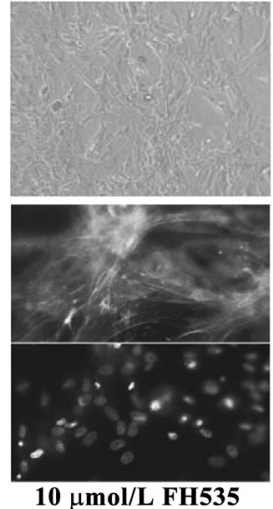

$10 \mu \mathrm{mol} / \mathrm{L}$ FH535

D

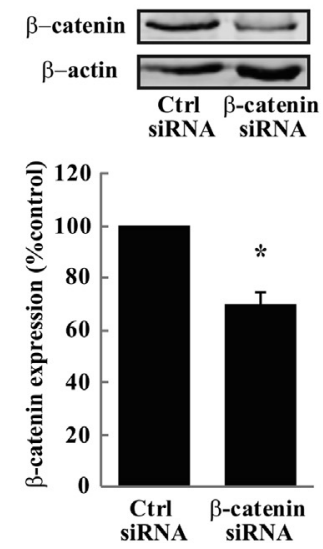

B
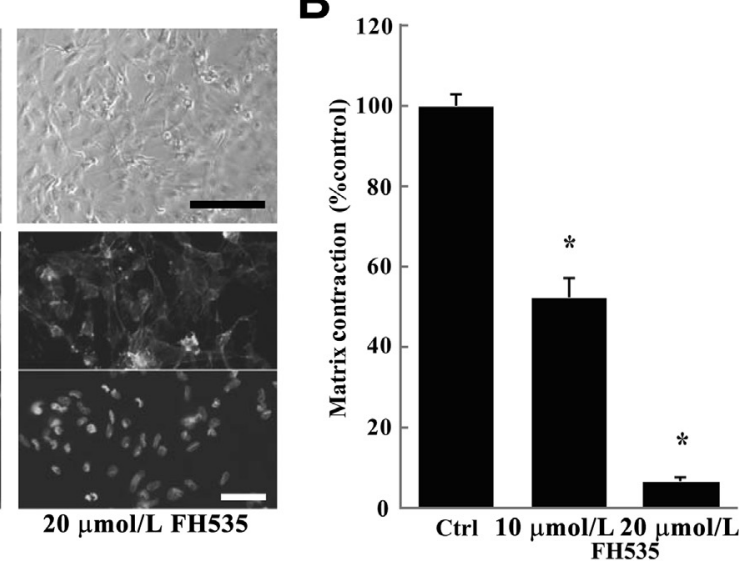

E

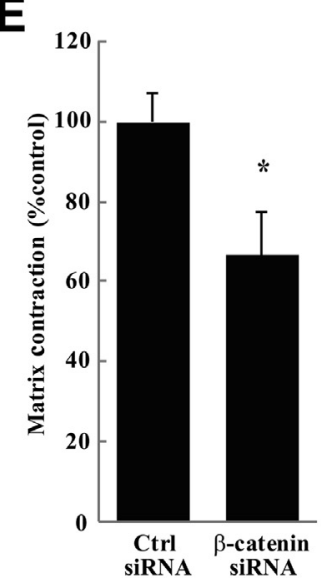

Figure 3 In vitro contractile membrane formation and the assessment of matrix contraction. A: Phase-contrast images and actin and nuclear staining of RPE-derived cells on type I collagen matrix after 3 days in culture. In the control condition, cells form a dense membrane with strong stress-fiber expression. Addition of FH535 prevented formation of a dense membrane in a concentration-dependent manner. B: Contraction of the collagen matrix after release from the plate. FH535 prevented gel contraction in a concentration-dependent manner. Values are normalized to control. C: The effect of GW9662, an analog of FH535 that inhibits PPAR signaling but not $\beta$-catenin signaling, on contractile membrane formation. GW9662 had little effect on dense membrane formation, and the contraction of collagen matrix was not significantly affected. D: Western blot analysis of $\beta$-catenin in cells treated with control or $\beta$-catenin siRNA. A significant knockdown in $\beta$-catenin protein expression was observed in $\beta$-catenin siRNA-treated cells, compared with control siRNA-treated cells. E: The effect of $\beta$-catenin knockdown on contractile membrane formation. Treatment with $\beta$-catenin siRNA resulted in formation of a less dense membrane, and the contraction of collagen matrix was significantly reduced. Data are expressed as means \pm SEM of 3 different samples (D), 8 different samples from three different batches $(\mathbf{E})$, or $\geq 12$ different samples from four different batches (B and $\mathbf{C})$. Scale bar $=50 \mu \mathrm{m}$.

formation of dense contractile membranes was further investigated using the connexon channel blocker CBX. Addition of CBX to the culture medium led to the failure of cells to form a dense membrane and significantly inhibited matrix contraction in a concentration-dependent manner (Figure 4C). When CBX was added after formation of the contractile membrane on day 3 , it had no significant effect on collagen matrix contraction (Figure 4D). Addition of FH535 to the dense contractile membrane significantly inhibited contraction, but to a lesser extent than with addition of FH535 before dense membrane formation (compare Figures 3B and 4D).

\section{Effects of $\beta$-Catenin Signaling Inhibition in Vivo}

To investigate whether PVR could be prevented, we tested inhibition of $\beta$-catenin signaling in vivo. Initially, the effect of FH535 on PVR formation was investigated using the in vivo swine PVR model. ${ }^{34}$ In this model, RPE cells injected into the vitreous cavity undergo EMT, adopt fibroblastic and myofibroblastic phenotypes, and form contractile membranes that results in TRD. ${ }^{34}$ Consistent with previous findings, in the absence of FH535 (control), all eyes except one (7/8) developed PVR with TRD ( $\geq$ grade 2 PVR). By contrast, none of the eyes injected with 20 $\mu \mathrm{mol} / \mathrm{L}$ FH535 (0/9) developed this complication (Figure 5A). Examination of enucleated control eyes (without FH535 treatment) revealed the presence of a dense fibrotic membrane on the surface of the detached retina (Figure 5B). Staining for GFP on paraffin-embedded sections from control eyes revealed multiple layers of cells in these dense membranes (Figure 5C). By contrast, although an epiretinal membrane of $\mathrm{GFP}^{+}$cells was observed in most eyes treated with FH535, these membranes were essentially 
A
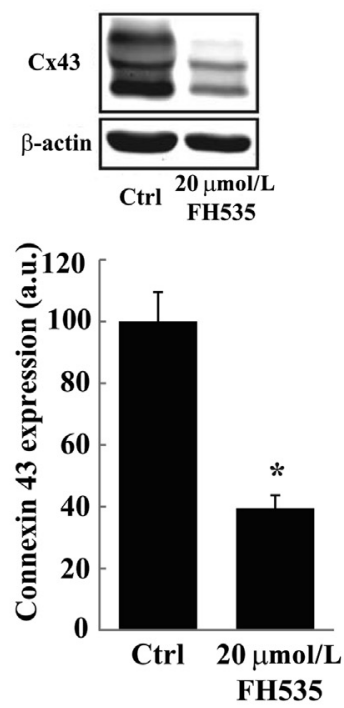

C

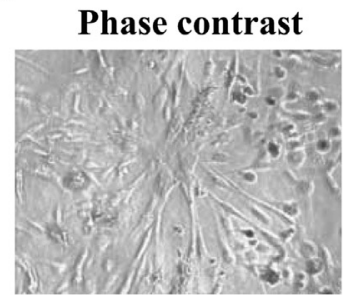

CBX $10 \mu \mathrm{mol} / \mathrm{L}$

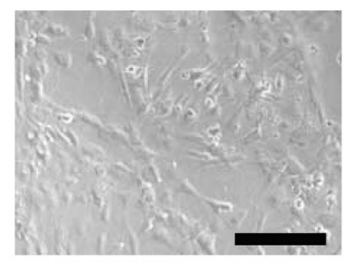

CBX $30 \mu \mathrm{mol} / \mathrm{L}$
B
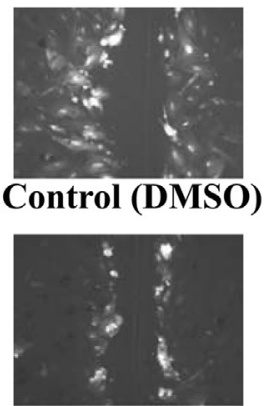

FH53510 $\mu \mathrm{mol} / \mathrm{L}$

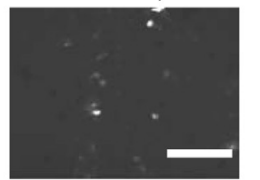

FH53520 $\mu \mathrm{mol} / \mathrm{L}$

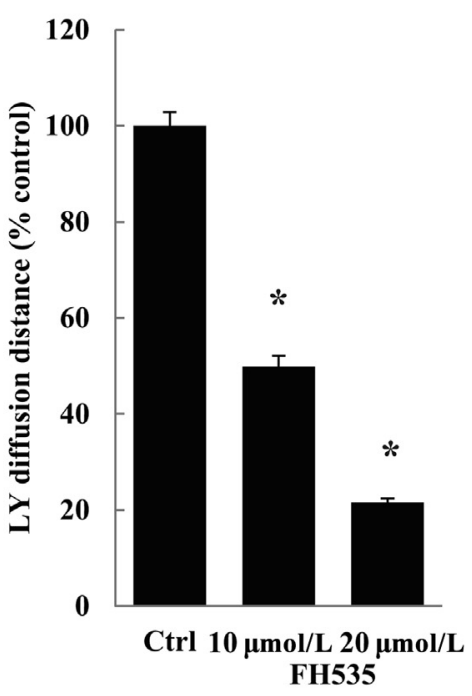

D

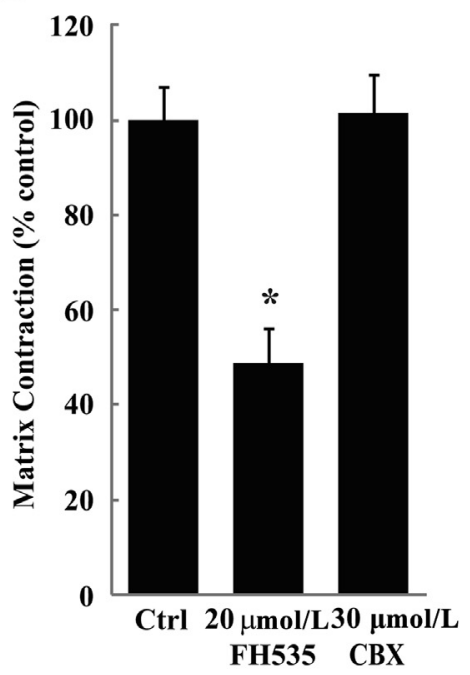

Figure 4 Connexin-43 is a target of $\beta$-catenin signaling and is involved in formation of the dense contractile membrane. A: Western blot analysis of connexin-43 expression of passage 3 RPE cells cultured in the presence or absence of FH535. FH535 (20 $\mu \mathrm{mol} / \mathrm{L})$ significantly reduced connexin- 43 expression. B: The effect of FH535 on gap junction function. The distance of Lucifer Yellow diffusion, a measure of gap junction function, was significantly reduced in FH535-treated cells compared with controls in a concentration-dependent manner. C: The effect of CBX, a connexon channel inhibitor, on contractile membrane formation. CBX prevented formation of a dense membrane and the resultant contraction of the collagen matrix was significantly reduced in a concentration-dependent manner. Matrix contraction values are normalized to control. D: Addition of CBX on day 3, after formation of a dense contractile membrane, did not prevent matrix contraction. By contrast, contraction was significantly reduced by FH535 even when added on day 3 . Data are expressed as means \pm SEM of 4 different samples (A), 9 different samples from three different batches (C and $\mathbf{D})$, or $\geq 12$ different samples from four different batches (B). ${ }^{*} P<0.05$ versus control. LY, Lucifer Yellow. Scale bar $=50 \mu \mathrm{m}$.

monolayers with only a few areas of focal density (Figure 5, $B$ and $C)$.

Next, we investigated the effect of $20 \mu \mathrm{mol} / \mathrm{L}$ FH535, administered twice a week by intravitreal injection, on electrophysiologic function of the retina. Indirect ophthalmoscopy revealed no clinical abnormality in either the anterior or posterior segment. Full-field electroretinographic analysis of eyes injected with FH535 revealed no changes relative to baseline values (before FH535 injection) or to control eyes injected with DMSO (Figure 6).

\section{Discussion}

Here, using both in vitro and in vivo models, we have demonstrated that $\beta$-catenin signaling is activated in RPE cells in EMT and that the signaling remains active in dedifferentiated RPE cells. Translocation of active $\beta$-catenin into the nucleus, which is required for the activation of the canonical Wnt pathway, ${ }^{38}$ was observed only in dedifferentiated cells, and not in the original differentiated cells cultured under the same condition (Figure 1A). We have previously shown that 
A

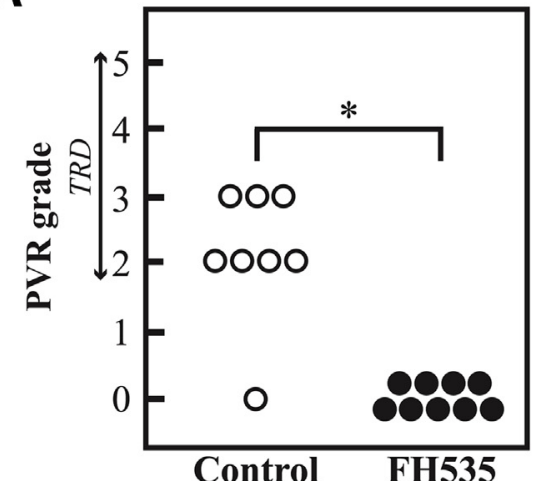

B

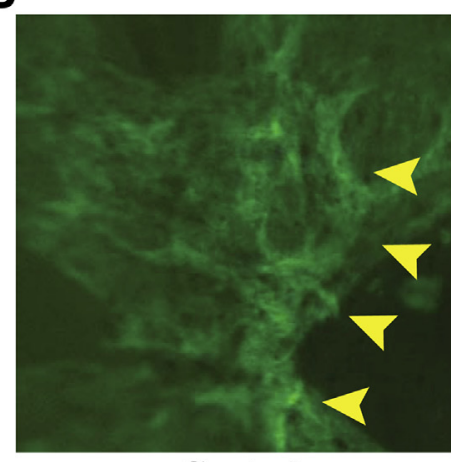

Control

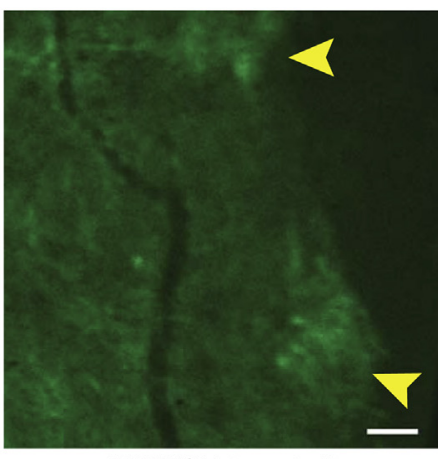

FH535 treated

C

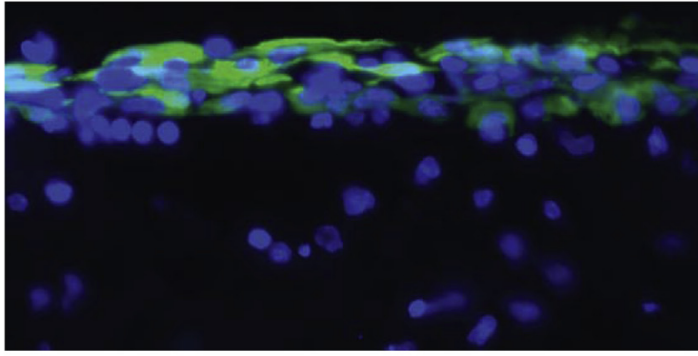

Control

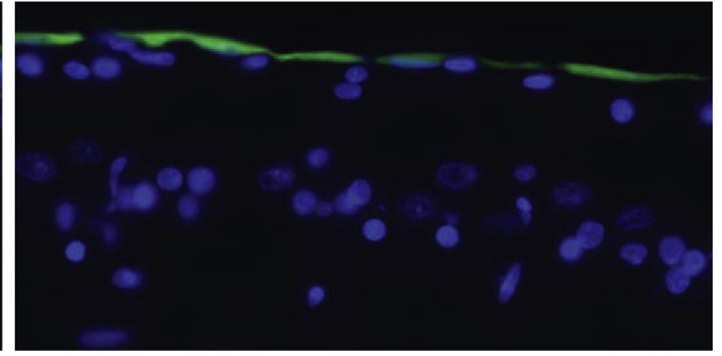

FH535 treated

Figure 5 Effect of FH535 on PVR in vivo. A: PVR grading on day 14 of eyes with or without FH535 treatment. Classification of PVR was based on the Silicone Study Classification System for PVR. ${ }^{36}$ Stage 0, normal retina, retinal or vitreous pigment clumps; stage 1, inner retina wrinkling; stage 2, retinal detachment (RD), one quadrant; stage 3, RD, two quadrants; stage 4, RD, three quadrants; stage 5, RD, four quadrants. TRD was observed in 7 of 8 eyes under the control condition (without FH535 treatment). No TRD was observed in any of the FH535-treated eyes (0 of 9). B: Membranes containing GFP ${ }^{+}$cells form on the surface of the retina. In control eyes, a dense fibrotic membrane with foci (arrowheads) was observed on the surface of the detached retina. Eyes treated with FH535 formed a membrane containing $\mathrm{GFP}^{+}$cells but with only isolated focal dense regions (arrowheads). C: Immunohistological assay of paraffin-embedded sections revealed dense fibrotic membranes on the surface of the detached retina in the control eye, consisting of multiple layers of GFP ${ }^{+}$cells. In eyes treated with FH535, the membranes consisted of a monolayer of cells. ${ }^{*} P<0.05$ versus control.

disruption of cadherin binding by EGTA is required to induce EMT and proliferation of differentiated RPE cells. ${ }^{16}$ A recent publication by Chen et $\mathrm{al}^{39}$ also demonstrated activation of $\beta$-catenin signaling only after disruption of cadherin binding by EGTA in a postconfluent ARPE-19 human RPE cell line. Thus, similar to other cell types, ${ }^{40}$ disruption of cadherin binding and the release of $\beta$-catenin is required for its role as a signaling molecule in RPE cells. The released $\beta$-catenin in the cytoplasm then needs to avoid destruction to translocate into the nucleus and activate signaling. GSK-3 $\beta$, a key component of the destruction mechanism, can be inactivated by Akt. ${ }^{40}$ Serum and vitreous fluid contain various molecules that can activate Akt in RPE cells. ${ }^{41-43}$ Stimulation of dedifferentiated RPE cells with either serum or vitreous fluid resulted in nuclear translocation of $\beta$-catenin and activation of signaling.

Dedifferentiated RPE cells start to migrate and proliferate both in vitro and in vivo. RPE cell proliferation is regulated by expression of cyclin D1, ${ }^{42,44}$ and cyclin D1 expression is regulated by $\beta$-catenin signaling. ${ }^{20}$ In the present study, inhibition of $\beta$-catenin signaling by FH535 led to reduced cyclin D1 expression and inhibited cell proliferation (Figure 1D and Figure 2, A and C).

Formation and contraction of the epiretinal membrane is a characteristic feature of PVR. Dense fibrotic membranes are normally present at the site of $\mathrm{TRD},{ }^{4,10}$ and according to Machemer, 10,p739 "it is this whole membrane that contracts" and results in TRD. We therefore performed an in vitro assay to study the formation and contraction of a fibrotic membrane. Under control conditions (without added drug),

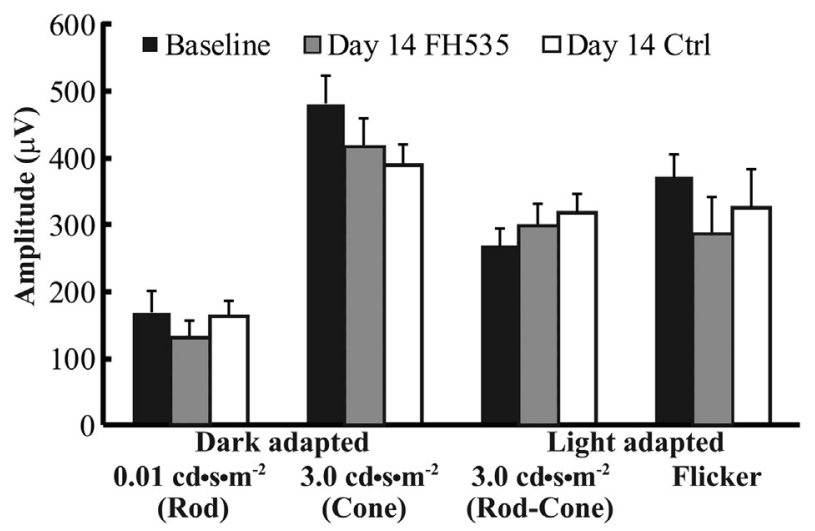

Figure 6 Effect of FH535 on electrophysiological function of the retina. Full-field electroretinography revealed no change in retinal function with FH535 treatment. The electroretinographic response in eyes treated for 14 days with FH535 was similar to that of baseline (before FH535 injection) and to that of DMSO-injected control eyes on day 14. Data are expressed as means \pm SEM. $n=5$ per group. 
a dense fibrotic membrane derived from RPE cells formed on the collagen matrix, and the matrix contracted rapidly on release. Membranes did form when $\beta$-catenin signaling was inhibited by FH535, but these membranes were not as dense as in the control and were significantly less contractile. Similarly, when FH535 was administered to eyes in vivo, epiretinal membranes did form, but these membranes were not dense and did not cause TRD in our swine PVR model (in contrast to control eyes). Thus, both in vitro and in vivo assays demonstrate that dense and strongly contractile fibrotic membranes are required for the development of TRD experimentally, similar to that observed clinically. ${ }^{4,9}$

Our data also indicate that connexin-43, a downstream target of $\beta$-catenin signaling, ${ }^{37,45}$ plays a role in formation of dense contractile membranes. Not only was expression of connexin43 significantly reduced by FH535 treatment, but functional inhibition of connexon channels by CBX also led to inhibition of the formation of the dense contractile membrane. In addition to its use as a gap junction and hemichannel inhibitor, CBX has also been widely used as an inhibitor of $11 \beta$-hydroxysteroid dehydrogenase. ${ }^{46}$ However, the effect of CBX on $11 \beta$ hydroxysteroid dehydrogenase plateaus at $\leq 10 \mu \mathrm{mol} / \mathrm{L},{ }^{47-50}$ and because the inhibition of collagen matrix contraction between 10 and $30 \mu \mathrm{mol} / \mathrm{L} \mathrm{CBX}$ was significantly different in the present study, we believe that the inhibitory effect we observed

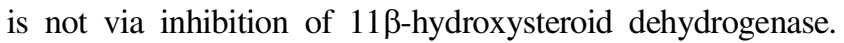
Interestingly, CBX did not prevent in vitro contraction of the dense membrane once it had formed. This suggests that connexins play a greater role early during maturation of the fibrotic membrane and a lesser role in contraction once the fibrotic membrane is established. Connexins (and the most extensively studied connexin- 43 in particular) have been shown to regulate a variety of cell functions. ${ }^{51,52}$ In addition to their wellestablished role as a gap junction channel component, it has recently been reported that connexins may function as cell-cell adhesion molecules and as regulators of cell migration..$^{53-55}$ Connexins could thus also be involved in maturation of the dense fibrotic membrane. Alternatively, gap junctional intercellular communication could play a role in fibrotic changes, through myofibroblast differentiation as well as collagen and fibronectin deposition. ${ }^{56,57}$ Further studies will be required to identify the specific role or roles of connexin- 43 in formation of fibrotic membranes.

The effect of FH535 in vivo was investigated up to 14 days after the induction of PVR, because our model was developed to investigate the early phase of this disease. Our in vitro and in vivo data suggest that the role of RPE-derived cells in formation of a dense fibrotic membrane in early PVR causing TRD can be obviated if FH535 is injected into the vitreous cavity. We do not know the pharmacodynamics of intravitreal FH535, and therefore used repeated intravitreal injection. Continued administration of this drug by an intravitreal slow-release drug device may be required in a clinical setting for prevention of PVR for longer periods. Although RPE cells play an important role during the early stages of PVR, ${ }^{4,13-15}$ glial cells are considered to be a major cell type during later stages. Wnt/ $\beta$-catenin signaling plays a role in proliferation of glial cells after injury, ${ }^{58,59}$ and inhibitors of this signaling pathway would likely be beneficial in inhibiting the growth and spread of this cell type as well. Further studies will be required to determine whether proliferation and contraction by glia-derived cells can similarly be prevented by $\beta$-catenin signaling inhibitors.

In summary, we have demonstrated that $\beta$-catenin signaling is involved in PVR-related RPE cell functions, namely, EMT, migration, proliferation, and formation of the contractile membrane. Connexin-43, a downstream target of $\beta$-catenin signaling, also plays a role in formation of contractile membranes. Inhibition of $\beta$-catenin signaling with inhibitors such as FH535 may be a viable option for the prevention of PVR, because such inhibition blocked development of TRD in our PVR model without adverse effect on retinal electrophysiology. Further studies investigating the safety of such drugs are worth undertaking.

\section{References}

1. Rosenbloom J, Mendoza FA, Jimenez SA: Strategies for anti-fibrotic therapies. Biochim Biophys Acta 2012, 1832:1088-1103

2. The classification of retinal detachment with proliferative vitreoretinopathy. Ophthalmology 1983, 90:121-125

3. Cardillo JA, Stout JT, LaBree L, Azen SP, Omphroy L, Cui JZ, Kimura H, Hinton DR, Ryan SJ: Post-traumatic proliferative vitreoretinopathy. The epidemiologic profile, onset, risk factors, and visual outcome. Ophthalmology 1997, 104:1166-1173

4. Pastor JC, de la Rúa ER, Martín F: Proliferative vitreoretinopathy: risk factors and pathobiology. Prog Retin Eye Res 2002, 21:127-144

5. Hiscott P, Sheridan C, Magee RM, Grierson I: Matrix and the retinal pigment epithelium in proliferative retinal disease. Prog Retin Eye Res 1999, 18:167-190

6. Nagasaki H, Shinagawa K, Mochizuki M: Risk factors for proliferative vitreoretinopathy. Prog Retin Eye Res 1998, 17:77-98

7. Oberstein SY, Byun J, Herrera D, Chapin EA, Fisher SK, Lewis GP: Cell proliferation in human epiretinal membranes: characterization of cell types and correlation with disease condition and duration. Mol Vis 2011, 17:1794-1805

8. Charteris DG, Sethi CS, Lewis GP, Fisher SK: Proliferative vitreoretinopathy-developments in adjunctive treatment and retinal pathology. Eye 2002, 16:369-374

9. Bringmann A, Wiedemann P: Involvement of Müller glial cells in epiretinal membrane formation. Graefes Arch Clin Exp Ophthalmol 2009, 247:865-883

10. Machemer R: Pathogenesis and classification of massive periretinal proliferation. Br J Ophthalmol 1978, 62:737-747

11. Guidry C: The role of Müller cells in fibrocontractive retinal disorders. Prog Retin Eye Res 2005, 24:75-86

12. Saika S, Yamanaka O, Flanders KC, Okada Y, Miyamoto T, Sumioka T, Shirai K, Kitano A, Miyazaki K, Tanaka S, Ikeda K: Epithelial-mesenchymal transition as a therapeutic target for prevention of ocular tissue fibrosis. Endocr Metab Immune Disord Drug Targets 2008, 8:69-76

13. Morino I, Hiscott P, McKechnie N, Grierson I: Variation in epiretinal membrane components with clinical duration of the proliferative tissue. Br J Ophthalmol 1990, 74:393-399

14. Yamashita H, Hori S, Masuda K: Population and proportion of component cells in preretinal membranes. Jpn J Ophthalmol 1986, 30:269-281

15. Campochiaro PA: Pathogenic mechanisms in proliferative vitreoretinopathy. Arch Ophthalmol 1997, 115:237-241 
16. Tamiya S, Liu L, Kaplan HJ: Epithelial-mesenchymal transition and proliferation of retinal pigment epithelial cells initiated upon loss of cell-cell contact. Invest Ophthalmol Vis Sci 2010, 51:2755-2763

17. Kalluri R, Weinberg RA: The basics of epithelial-mesenchymal transition [Erratum appeared in J Clin Invest 2010, 120:1786]. J Clin Invest 2009, 119:1420-1428

18. Casaroli-Marano RP, Pagan R, Vilaró S: Epithelial-mesenchymal transition in proliferative vitreoretinopathy: intermediate filament protein expression in retinal pigment epithelial cells. Invest Ophthalmol Vis Sci 1999, 40:2062-2072

19. Grisanti S, Guidry C: Transdifferentiation of retinal pigment epithelial cells from epithelial to mesenchymal phenotype. Invest Ophthalmol Vis Sci 1995, 36:391-405

20. Morin PJ: beta-Catenin signaling and cancer. Bioessays 1999, 21: $1021-1030$

21. Lam AP, Gottardi CJ: $\beta$-Catenin signaling: a novel mediator of fibrosis and potential therapeutic target. Curr Opin Rheumatol 2011, 23:562-567

22. Handeli S, Simon JA: A small-molecule inhibitor of Tcf/beta-catenin signaling down-regulates PPARgamma and PPARdelta activities. Mol Cancer Ther 2008, 7:521-529

23. Vaid M, Prasad R, Sun Q, Katiyar SK: Silymarin targets beta-catenin signaling in blocking migration/invasion of human melanoma cells. PloS One 2011, 6:e23000

24. Umazume K, Liu L, Scott PA, Fernandez de Castro JP, McDonald K, Kaplan HJ, Tamiya S: Inhibition of PVR with a tyrosine kinase inhibitor, dasatinib, in the swine. Invest Ophthalmol Vis Sci 2013, 54: $1150-1159$

25. van Noort M, Meeldijk J, van der Zee R, Destree O, Clevers H: Wnt signaling controls the phosphorylation status of beta-catenin. J Biol Chem 2002, 277:17901-17905

26. Liang CC, Park AY, Guan JL: In vitro scratch assay: a convenient and inexpensive method for analysis of cell migration in vitro. Nat Protoc 2007, 2:329-333

27. Parapuram SK, Chang B, Li L, Hartung RA, Chalam KV, NairMenon JU, Hunt DM, Hunt RC: Differential effects of TGFbeta and vitreous on the transformation of retinal pigment epithelial cells. Invest Ophthalmol Vis Sci 2009, 50:5965-5974

28. Grinnell F, Petroll WM: Cell motility and mechanics in three-dimensional collagen matrices. Annu Rev Cell Dev Biol 2010, 26:335-361

29. Grinnell F, Ho CH, Lin YC, Skuta G: Differences in the regulation of fibroblast contraction of floating versus stressed collagen matrices. J Biol Chem 1999, 274:918-923

30. Morales SA, Mareninov S, Prasad P, Wadehra M, Braun J, Gordon LK: Collagen gel contraction by ARPE-19 cells is mediated by a FAK-Src dependent pathway. Exp Eye Res 2007, 85:790-798

31. Meitinger D, Hunt DM, Shih DT, Fox JC, Hunt RC: Vitreousinduced modulation of integrins in retinal pigment epithelial cells: effects of fibroblast growth factor-2. Exp Eye Res 2001, 73:681-692

32. el-Fouly MH, Trosko JE, Chang CC: Scrape-loading and dye transfer. A rapid and simple technique to study gap junctional intercellular communication. Exp Cell Res 1987, 168:422-430

33. Sonoda S, Spee C, Barron E, Ryan SJ, Kannan R, Hinton DR: A protocol for the culture and differentiation of highly polarized human retinal pigment epithelial cells. Nat Protoc 2009, 4:662-673

34. Umazume K, Barak Y, McDonald K, Liu L, Kaplan HJ, Tamiya S: Proliferative vitreoretinopathy in the swine-a new model. Invest Ophthalmol Vis Sci 2012, 53:4910-4916

35. Sanchez I, Martin R, Ussa F, Fernandez-Bueno I: The parameters of the porcine eyeball. Graefes Arch Clin Exp Ophthalmol 2011, 249: 475-482

36. Machemer R, Aaberg TM, Freeman HM, Irvine AR, Lean JS, Michels RM: An updated classification of retinal detachment with proliferative vitreoretinopathy. Am J Ophthalmol 1991, 112:159-165

37. van der Heyden MA, Rook MB, Hermans MM, Rijksen G, Boonstra J, Defize LH, Destrée OH: Identification of connexin43 as a functional target for Wnt signalling. J Cell Sci 1998, 111:1741-1749
38. MacDonald BT, Tamai K, He X: Wnt/beta-catenin signaling: components, mechanisms, and diseases. Dev Cell 2009, 17:9-26

39. Chen HC, Zhu YT, Chen SY, Tseng SC: Wnt signaling induces epithelial-mesenchymal transition with proliferation in ARPE-19 cells upon loss of contact inhibition. Lab Invest 2012, 92:676-687

40. Valenta T, Hausmann G, Basler K: The many faces and functions of $\beta$-catenin. EMBO J 2012, 31:2714-2736

41. Lei H, Rhéaume MA, Velez G, Mukai S, Kazlauskas A: Expression of PDGFR $\alpha$ is a determinant of the PVR potential of ARPE19 cells. Invest Ophthalmol Vis Sci 2011, 52:5016-5021

42. Parrales A, López E, López-Colomé AM: Thrombin activation of $\mathrm{PI} 3 \mathrm{~K} / \mathrm{PDK} 1 / \mathrm{Akt}$ signaling promotes cyclin D1 upregulation and RPE cell proliferation. Biochim Biophys Acta 2011, 1813:1758-1766

43. Pennock S, Rhéaume MA, Mukai S, Kazlauskas A: A novel strategy to develop therapeutic approaches to prevent proliferative vitreoretinopathy. Am J Pathol 2011, 179:2931-2940

44. Hecquet C, Lefevre G, Valtink M, Engelmann K, Mascarelli F: Activation and role of MAP kinase-dependent pathways in retinal pigment epithelial cells: ERK and RPE cell proliferation. Invest Ophthalmol Vis Sci 2002, 43:3091-3098

45. Oyamada M, Takebe K, Oyamada Y: Regulation of connexin expression by transcription factors and epigenetic mechanisms. Biochim Biophys Acta 2013, 1828:118-133

46. Diederich S, Quinkler M, Burkhardt P, Grossmann C, Bahr V, Oelkers W: 11Beta-hydroxysteroid-dehydrogenase isoforms: tissue distribution and implications for clinical medicine. Eur J Clin Invest 2000, 30(Suppl 3):21-27

47. Cho YS, Kim CH, Cheon HG: Cell-based assay for screening 11 betahydroxysteroid dehydrogenase 1 inhibitors. Anal Biochem 2009, 392: $110-116$

48. Gomez-Sanchez EP, Cox D, Foecking M, Ganjam V, GomezSanchez CE: 11 beta-hydroxysteroid dehydrogenases of the choriocarcinoma cell line JEG-3 and their inhibition by glycyrrhetinic acid and other natural substances. Steroids 1996, 61:110-115

49. Leckie C, Chapman KE, Edwards CR, Seckl JR: LLC-PK1 cells model 11 beta-hydroxysteroid dehydrogenase type 2 regulation of glucocorticoid access to renal mineralocorticoid receptors. Endocrinology 1995, 136:5561-5569

50. Patel FA, Sun K, Challis JR: Local modulation by 11 beta-hydroxysteroid dehydrogenase of glucocorticoid effects on the activity of 15 hydroxyprostaglandin dehydrogenase in human chorion and placental trophoblast cells. J Clin Endocrinol Metab 1999, 84:395-400

51. Saez JC, Berthoud VM, Branes MC, Martinez AD, Beyer EC: Plasma membrane channels formed by connexins: their regulation and functions. Physiol Rev 2003, 83:1359-1400

52. Solan JL, Lampe PD: Connexin 43 phosphorylation: structural changes and biological effects. Biochem J 2009, 419:261-272

53. Bao B, Jiang J, Yanase T, Nishi Y, Morgan JR: Connexon-mediated cell adhesion drives microtissue self-assembly. FASEB J 2011, 25: $255-264$

54. Cotrina ML, Lin JH, Nedergaard M: Adhesive properties of connexin hemichannels. Glia 2008, 56:1791-1798

55. Kameritsch P, Pogoda K, Pohl U: Channel-independent influence of connexin 43 on cell migration. Biochim Biophys Acta 2012, 1818: 1993-2001

56. Ehrlich HP, Diez T: Role for gap junctional intercellular communications in wound repair. Wound Repair Regen 2003, 11:481-489

57. Ehrlich HP, Sun B, Saggers GC, Kromath F: Gap junction communications influence upon fibroblast synthesis of type I collagen and fibronectin. J Cell Biochem 2006, 98:735-743

58. Liu B, Hunter DJ, Rooker S, Chan A, Paulus YM, Leucht P, Nusse Y, Nomoto H, Helms JA: Wnt signaling promotes Müller cell proliferation and survival after injury. Invest Ophthalmol Vis Sci 2013, 54:444-453

59. White BD, Nathe RJ, Maris DO, Nguyen NK, Goodson JM, Moon RT, Horner PJ: beta-Catenin signaling increases in proliferating $\mathrm{NG} 2+$ progenitors and astrocytes during post-traumatic gliogenesis in the adult brain. Stem Cells 2010, 28:297-307 